\title{
Impactos de lo global en lo local: Gentrificación en ciudades latinoamericanas
} Impacts of the global into the local: Gentrification in Latin American cities

Gabriel Gómez Carmona, Alberto Javier Villar Calvo

\section{Filiación}

UAEM, México

E mail: gabocop28@gmail.com, betovillardf@gmail.com

Primera versión recibida en: 07 de mayo, 2015

Última versión recibida en: 25 de junio, 2015

\section{Resumen}

La realidad global que actualmente viven las ciudades implica una recomposición de las relaciones socio-espaciales y los tradicionales patrones de convivencia y consumo. La ciudad toma un rol determinante en lo político, económico y social al buscar su incorporación dentro de la dinámica globalizante, lo cual crea la existencia de diversas zonas al interior de la ciudad. El artículo aborda de manera general los procesos de revitalización de las zonas centrales de dos ciudades latinoamericanas (Santiago y Ciudad de México) y sus cambios socio-espaciales que se generan. Para el caso latinoamericano, la evidencia muestra que la revaloración y rehabilitación de centros históricos y barrios coloniales para crear mejores lugares de tránsito, ocio y vida para sus habitantes, implican una fuerte transformación de los mismos, con realidades dispares, fragmentación urbana, segregación y gentrificación.

\section{Palabras Clave}

Gentrificación; centros históricos; patrimonio

\begin{abstract}
The global reality now living cities is a restructuring of the socio-spatial relations and traditional patterns of living and consumption. The city takes a decisive role in the political, economic and social to seek their incorporation into the globalizing dynamic, which creates the existence of different areas inside the city. The article discusses in general terms the processes of revitalization of the central areas of two Latin American cities (Santiago and Mexico City) and the socio-spatial changes that are generated. For the Latin American case, the evidence shows that the revaluation and rehabilitation of historic centers and colonial neighborhoods to create better places of transit, leisure and life for their inhabitants, involving heavy transformation thereof, with disparate realities, urban fragmentation and segregation gentrification.
\end{abstract}

\section{Key Words}

Gentrification; historic centers; heritage

\section{Sumario}

Introducción.

1 Desarrollo

1.1 El Escenario Latinoamericano.

1.2 ¿Gentrificación en Centros Históricos?

2 Casos de Estudio

2.1 El caso Santiago, Chile.

2.2 El caso Ciudad de México (CDMX).

3 Lugares Heterogéneos: Zonas de Encuentro e Identidad.

4 Conclusiones

Bibliografía 


\section{Introducción.}

\section{El Escenario Global.}

La realidad que actualmente viven las ciudades implica fuertes y dinámicos procesos de cambio caracterizados por una recomposición de las relaciones socio-espaciales y los tradicionales patrones de convivencia y consumo en un mundo globalizado.

Los cambios experimentados en las últimas cuatro décadas en el modelo económico dominante, ha generado un transformación sin precedentes en lo productivo, político, social y cultural a nivel planetario, repercutiendo directamente en la forma de construir, vivir y socializar el espacio urbano. Además, esto ha llevado a que las ciudades concentren actualmente la mayor cantidad de población a nivel mundial, teniendo que para el 2014 , el $54 \%$ de la población mundial residía ya en áreas urbanas, y estimándose que para el 2050 la cifra alcance un $66 \%{ }^{1}$.

Desde este escenario, observamos que este mundo globalizado requiere de las ciudades como lugares estratégicos para su promoción y proyección planetaria (Carrión, 2001). Es por ello, que éstas experimentan fuertes procesos de transformación socio-espacial y el surgimiento de nuevas dinámicas urbanas que nos cuestionan si estos cambios implican una ruptura con el modelo de ciudad que se venía construyendo, o si por el contrario, representa una continuidad y una radicalización del modelo de ciudad desarrollista (De Mattos, 1999).

Hoy percibimos cómo la ciudad toma un rol importante en lo político y económico superando su propio ámbito de existencia, lo local, dando paso a lo glocal, que nos habla de esa nueva relación complementaria entre lo global y lo local pues, así como la globalización requiere de lo local para existir, lo local necesita de lo global para desarrollarse (Carrión, 2001: 10-11). Las ciudades latinoamericanas no son ajenas a esto, hoy experimentan dichos procesos pero con características que las asemejan o distinguen de otras realidades planetarias o regionales.

Es así que las ciudades buscan su incorporación a la dinámica del mundo global, para ello, su éxito y vigencia está en función de articularse en red con la generación de condiciones de competitividad y posicionamiento (Carrión, 2001: 11). Se habla de ciudades ganadoras o perdedoras, con base a sus influencias, desarrollo, tamaño, o bien, el dominio y acaparamiento que ejercen sobre las inversiones, los recursos, servicios, e infraestructur. Desde esta perspectiva, se distinguen ciudades globales (Tokio, Nueva York, Londres, ) y ciudades regionales (Ciudad de México, Sao Paulo, Santiago) (García Canclini, 2002).

Estos procesos llevan a una fuerte terciarización de la economía generando transformaciones y nuevas formas de urbanización de la ciudad. De ahí la actual coexistencia al interior de las ciudades de: a) Zonas tugurizadas y marginadas; b) Zonas periurbanizadas, conectadas con la ciudad y con servicios; y c) Zonas centrales renovadas y gentrificadas (Vergara, 2013: 221). Estas dinámicas y los cambios en los patrones de convivencia, consumo y apropiación de los espacios urbanos producen en las ciudades, en nuestro caso, latinoamericanas, el surgimiento de diversas problemáticas sociales al interior de ellas, expresadas en procesos de elitización y segregación socio-espacial e, incluso, de gentrificación.

\section{Desarrollo}

\subsection{El Escenario Latinoamericano.}

El artículo aborda de manera general, los procesos de revitalización y revaloración de los centros históricos de dos ciudades latinoamericanas (Santiago y Ciudad de México), y los cambios socioespaciales que éstos conllevan dado que, para el caso latinoamericano, la evidencia muestra que

1 http://www.un.org/spanish/News/story.asp?NewsID=29935\#.VQJNZ9KG-So 
la revaloración y rehabilitación de centros históricos y barrios coloniales para crear mejores lugares de tránsito, ocio y vida para sus habitantes, implican una fuerte transformación de los mismos, con realidades sociales dispares, fragmentación urbana, segregación y por consecuencia, gentrificación.

Realizaremos una exploración general a la forma en la que estos procesos de transformación socio-espacial se han dado en estas dos ciudades latinoamericanas a partir del siguiente cuestionamiento: ¿existen procesos de gentrificación en los centros históricos de estas ciudades latinoamericanas como consecuencia de los programas de rehabilitación, renovación, revaloración urbana y repoblamiento, implementados en las dos últimas décadas para crear en éstos, mejores lugares de tránsito, ocio y vivienda para sus habitantes? El artículo se sustenta en un análisis de datos estadísticos que permiten ver el crecimiento poblacional y territorial de ambas ciudades en el último siglo, junto con los datos recabados en etnografías urbanas realizadas en ellas, lo que permitió contrastar vivencialmente su espacio urbano con el debate teórico existente y efectuar una aproximación a dichas realidades. Por tal motivo, las conclusiones expuestas no son definitivas, por el contrario, buscan propiciar nuevas líneas de discusión y análisis de éstas y otras experiencias para profundizar en el debate teórico de la realidad latinoamericana desde Latinoamérica.

Estamos convencidos que el estudio de dichos fenómenos permite tener una radiografía de la realidad socio-espacial que actualmente se vive en las ciudades latinoamericanas, principalmente en sus centros históricos patrimoniales, facilitando la comprensión de las causas y efectos de estos procesos de cambio, además de constituir potenciales herramientas para el análisis y toma de decisiones al interior de las ciudades y enriquecer el debate teórico actual sobre la realidad de nuestras ciudades.

En las últimas dos décadas los centros históricos y los barrios centrales de diversas ciudades latinoamericanas (Ciudad de México, Santiago, Quito, Buenos Aires, Montevideo, entre otras) han sido objeto de diversas intervenciones urbanas, buscando su rehabilitación y revaloración con fines de mejora en su imagen, conservación de su Patrimonio (urbano-arquitectónico) y mejores condiciones de tránsito, ocio y vida para sus usuarios y habitantes. Sin embargo, dichos procesos de rehabilitación tienen un trasfondo que va más allá de la simple mejora física de los espacios y las edificaciones, desembocando en una discusión sobre los pros y contras de estos procesos: políticas públicas aplicadas, intereses económicos subyacentes y los costos sociales o culturales que conllevan.

Uno de los aspectos abordados en el debate teórico es ese regreso a la ciudad construida (Carrión, 2001; Díaz-Parra, 2013), lo que habla de un proceso inverso a la expansión urbana y la periferización (Carrión, 2001), lo que Contreras (2011) denomina movilidad centrípeta o la densificación de espacios centrales. Pero, además este proceso se liga con planes de renovación concebidos desde un enfoque postindustrial que buscan la reconversión productiva de las ciudades, con el fin de obtener beneficios de la puesta en valor de su potencial y condiciones de centralidad (Vergara, 2013: 222) y, con ello, la incorporación a la dinámica global, siendo competitivas y atractivas para la inversión, los servicios, el comercio y el turismo.

\section{2 ¿Gentrificación en Centros Históricos?}

Lo anterior abre el debate sobre los principales usos y actividades que históricamente han albergado los centros fundacionales de las ciudades latinoamericanas (gobierno, cultura, vivienda, comercio, servicios), desde la época colonial y hasta nuestros días. Ello, lentamente llevó a una degradación y obsolescencia de numerosas edificaciones y por extensión, del Patrimonio urbano-arquitectónico, razón por la cual, la renovación de los centros históricos y los barrios centrales, cobra interés como objeto de estudio en la investigación urbana, por los 
programas y políticas públicas aplicadas en su rehabilitación, y las consecuentes transformaciones espaciales, económicas, culturales y sociales que generan estos cambios.

Por ello, centramos nuestro interés en este conjunto de transformaciones porque nos hablan de manera particular de la irrupción de procesos de elitización y gentrificación estimulados por el cambio socio-espacial de zonas históricas degradadas de la ciudad. Principalmente, a través de la inversión de capital público y mayoritariamente privado, para mejorarlas y obtener mayores ganancias a través de nuevos mercados inmobiliarios y nichos de consumo; la pérdida o expulsión de la población que tradicionalmente los habitaba por una elevación en el costo de la nueva vivienda; el cambio de uso de los edificios mejor conservados y en su caso, la renovación de las edificaciones existentes por otras que modifican la estructura e imagen urbana (alturas, estilos, materiales); la turistización y comercialización de la Cultura y el Patrimonio, además de cambios socio-económicos en la composición de los nuevos habitantes y usuarios de las zonas renovadas (medianos y altos ingresos) (De Mattos, 1999; Carrión, 2001; Díaz-Parra, 2013; Vergara, 2013).

Es así que la renovación o el rescate de los centros históricos implica más allá del simple uso de estos términos, acciones que buscan renovar no solo las edificaciones sino, los usos que tradicionalmente se le había dado a los centros históricos y barrios centrales, y rescatarlos de aquellos usos "indeseables" (tugurización, vivienda degradada, migrantes pobres, comercio informal, inseguridad, etc.) para potencializar económicamente los privilegios de su localización (disponibilidad de infraestructura, equipamiento, existencia de Patrimonio con la enorme carga simbólica de los mismos).

Recordemos que numerosos centros y barrios históricos latinoamericanos entraron en una fase de decaimiento y degradación desde la primera mitad del siglo XX, producto entre otros, de la salida de población de medianos y altos ingresos hacia los nuevos suburbios característicos de la ciudad fordista (Gómez-Carmona y Villar, 2013b) que significaron la puesta en marcha de los postulados del urbanismo moderno-le Corbusiano en la ciudad. Este proceso dio paso a que las viviendas desocupadas de las zonas centrales fueran habitadas por los nuevos migrantes campociudad en buscaron de nuevas condiciones de vida en una naciente fase de industrialización como fue el caso de la Ciudad de México. En esta ciudad, numerosos inmuebles dieron cabida a estos habitantes a través de viviendas colectivas en alquiler (vecindades o conventillos) resultado del fraccionamiento de las residencias burguesas.

De esta forma, hacia la década de los 50, el centro histórico de la Ciudad de México alcanzó su máxima concentración de población, posterior al congelamiento de rentas, fenómeno señalado como una de las principales causas de la desinversión y deterioro de los viejos inmuebles de las áreas centrales (Díaz-Parra, 2014: 3), aunque hacia la década de los 80, inicia su fase de despoblamiento incrementada por los sismos de 1985. Sin embargo, aún persisten estos fenómenos en diversas zonas de los centros y barrios históricos de ciudades latinoamericanas, como es el caso de Santiago de Chile, donde algunos sectores del centro histórico acogen a grupos específicos de migrantes latinoamericanos (como es el caso de los peruanos) y que se caracterizan no sólo por la tugurización de la vivienda colectiva sino, por una extensa red de comercio y servicios para satisfacer sus necesidades particulares (Torres e Hidalgo, 2009; Contreras, 2011; Borsdorf e Hidalgo, 2012), situación que con el paso del tiempo ha generado por otro lado, procesos de transculturación e hibridación cultural.

Es en este contexto que desde la década de los 80 del siglo pasado, comienzan a darse las primeras propuestas de revaloración de centros históricos en Latinoamérica. Así, hacia 1980 se delimita un perímetro de protección de monumentos en el centro histórico de la Ciudad de México (CDMX) y en 1987 es declarado Patrimonio Cultural de la Humanidad por la UNESCO, dando paso desde 1990, a diversos proyectos que han tendido a su rehabilitación, revitalización y repoblamiento, coincidiendo con la puesta en marcha de políticas neoliberales, la afluencia de 
capitales e inversiones extranjeras y el desarrollo de nuevas centralidades en la ciudad (DíazParra, 2014: 4).

Pero, ¿Qué motiva el regreso a la ciudad construida? ¿Qué motiva la rehabilitación de los centros históricos? Por lo menos, desde 1990 se dio una fuerte revaloración de las zonas centrales como lugares potenciales de inversión privada por las grandes ventajas que ofrece su localización, servicios, infraestructura, equipamiento, Patrimonio (histórico, urbano-arquitectónico, cultural, simbólico) (Gómez-Carmona y Villar, 2013a) y su latente capacidad como opción residencial (Contreras, 2011), lo que nos habla de una urbanización del territorio configurado, es decir, una urbanización de lo urbano (Carrión, 2001).

Asi también, ese regreso a las zonas centrales nos habla también de nuevas dinámicas económicas, de una articulación entre lugar de trabajo y residencia, estudios y residencia, y de un atractivo modo de vida urbano que hoy se oferta valorando las ventajas y riqueza (material y simbólica) de los centros y barrios históricos (Contreras, 2011), sin olvidar que diversos programas de rescate, rehabilitación y revaloración urbana, implicaron la eliminación o reubicación del comercio ambulante, la expulsión del pequeño y tradicional comercio, el incremento de la vigilancia y por tanto, la rehabilitación, reciclado o renovación de múltiples edificaciones con fines residenciales, dotacionales y de oficinas (Díaz-Parra, 2014). Y ello, de la mano de las declaratorias nacionales (Pueblos Mágicos para el caso de México) o internacionales (Patrimonio Cultural de la Humanidad-UNESCO) de los centros históricos como zonas con alto valor patrimonial.

En diversos casos esto impulsó fenómenos de revaloración y revitalización de las áreas centrales acompañados de un repoblamiento de las mismas por estratos sociales medios y medios-altos, atraídos por la puesta en marcha de distintivos andadores comerciales y culturales de la mano de programas culturales que buscan promover las ventajas propias de la zona y volver seductora la nueva oferta inmobiliaria promovida como un actual o moderno estilo de vida urbano.

En lo anterior, encontramos rasgos visibles de procesos de gentrificación de los centros y barrios históricos de las ciudades, situación que cobra interés por los altos costos sociales que estos fenómenos conllevan. Por ello debemos entender la gentrificación como un proceso que se manifiesta de diversas formas y con particulares características dependiendo del lugar y momento histórico en que se desarrolla pues, el querer ver la gentrificación desde el ámbito de la concepción original de R. Glass de 1964 para el ámbito inglés, nos lleva a un callejón sin salida que, lejos de permitir entender lo que hoy acontece, desemboca en discusiones estériles que nada aportan a la comprensión teórica de las actuales problemáticas urbanas que nos confrontan (Gómez-Carmona y Villar, 2013a).

Si bien, hoy podemos entender la gentrificación en términos generales como la elitización de ciertas áreas de la ciudad y la consecuente expulsión y segregación de la población de menores ingresos de estos espacios por población de mayores ingresos (Gómez-Carmona y Villar, 2013a), tampoco se puede afirmar que la manera en que se manifiesta el proceso en el ámbito anglosajón, donde se han realizado la mayoría de los estudios empíricos al respecto (Salinas 2013). Aun cuando la realidad anglosajona parece primar en su forma (Vergara, 2013), no se puede hablar de procesos idénticos para todas las realidades donde acontece, como tampoco es objetivo, leer el fenómeno de la misma manera como lo hizo R. Glass hace 50 años para la realidad inglesa y aplicarlo a la actual realidad latinoamericana dado que, el debate académico está dejando en claro que la gentrificación es un proceso de cambio socio-espacial que se presenta actualmente en diversas realidades urbanas planetarias, con características propias de cada lugar, que distinguen y quizá hacen única a cada una de ellas pero, teniendo como hilo conductor, términos que describen fenómenos como: rehabilitación, renovación, rescate urbano, 
elitización, aburguesamiento, fragmentación, polarización, segregación y exclusión socioespacial, tan solo por mencionar los más empleados.

Esto nos da idea de la magnitud que ha tenido la difusión no sólo del término sino, del proceso en un mundo globalizado en donde la constante, es una economía de libre mercado, políticas neoliberales y el achicamiento de las funciones del Estado, reflejado principalmente en el ámbito latinoamericano, en la desregulación, privatización y mercantilización del desarrollo urbano (Hidalgo y Arenas, 2011). De ahí la necesidad del estudio y documentación de la forma en la que se dan estos procesos de transformación socio-espacial en las ciudades latinoamericanas, permitiéndonos entender el modo en que procesos globales irrumpen e impactan en la realidad de un espacio local, o lo que es lo mismo, la forma en la que un fenómeno global se manifiesta en una realidad urbana específica.

Ahora presentaremos de manera breve la manera en la que estos procesos se han dado al interior de estas dos ciudades latinoamericanas, Santiago, Chile y Ciudad de México (CDMX), en un análisis que pone sobre la mesa de discusión, la existencia de gentrificación en los centros históricos de estas ciudades como consecuencia de los programas de rehabilitación, renovación urbana y repoblamiento.

\section{Casos de Estudio}

\subsection{El caso Santiago, Chile.}

Santiago es la capital de Chile y representa el principal centro urbano del país. El Área Metropolitana de Santiago (AMS), mejor conocida como Gran Santiago, está conformada por 37 comunas y forma parte de la Región Metropolitana de Santiago (RMS), que es una de las quince Regiones, en las que se divide Chile. La (RMS) se divide en 52 comunas y 6 provincias. Santiago a su vez, es la capital de la Provincia de Santiago, y una de las 32 comunas que la integran.

La población de la Provincia de Santiago ha crecido de la siguiente manera en el último siglo: 547 mil habitantes en 1907; 1.26 millones de habitantes en 1940; 3.8 millones de habitantes en 1982; 5.5 millones de habitantes en 2002; alcanzando para el 2012, una población de 6.05 millones de habitantes. Por su parte, la población de la Región Metropolitana de Santiago (RMS) ha sido: 4.32 millones de habitantes en 1982; 6.06 millones de habitantes en 2002; alcanzando en el 2012, una población de 6.70 millones de habitantes (Fig. 1), cifra que representa el 40\% del total de la población chilena que para ese mismo año se estimó en 17.44 millones de habitantes (Datos INE, 2014).

Los datos permiten ver el gran crecimiento urbano experimentado en Santiago, lo que la convierte en la principal y más grande urbe de esa región sudamericana, no exenta de complejos contrastes y problemáticas socio-espaciales, al grado de que para Greene y Soler (2004), la ciudad experimentó en las últimas décadas una rápida y explosiva transformación urbana por su nivel de concentración en el tiempo y el espacio, a diferencia de otras ciudades, además de que su estructura económica y social (al igual que otras ciudades latinoamericanas) se caracteriza por una fuerte segregación socio-espacial (p. 47,50). Los cambios observados en Santiago y su área metropolitana pueden entenderse como consecuencia de la dinámica de la reestructuración socio-económica aplicada posterior al golpe militar de 1973 y como efectos de la globalización sobre una metrópoli periférica principalmente en las últimas dos décadas (De Mattos, 1999). 


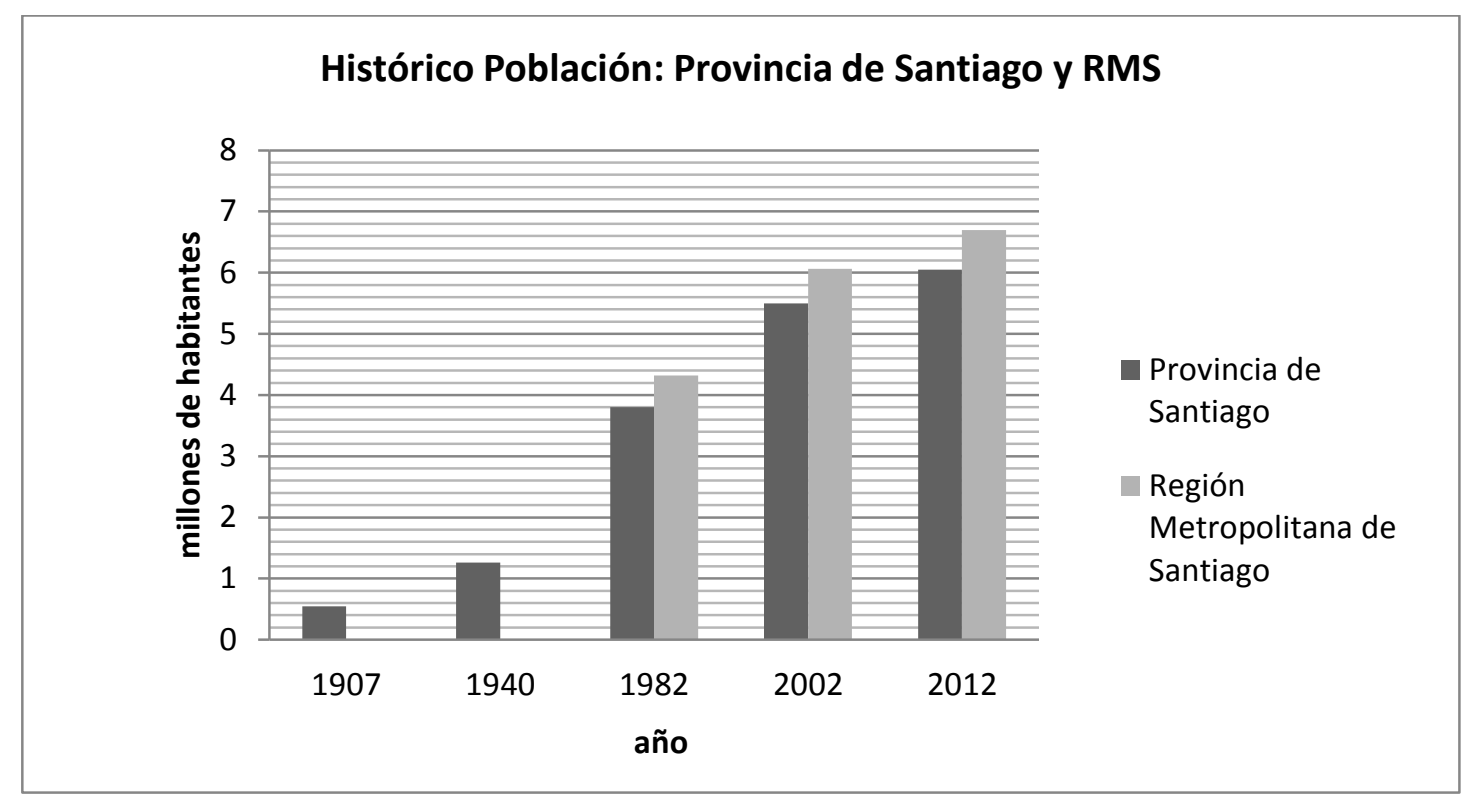

Fig. 1 Cuadro histórico de población para la Provincia de Santiago y la Región Metropolitana de Santiago (RMS)

Elaboración propia con datos de censos INE (1940, 1982, 2002, 2014).

Apreciamos que el Gran Santiago vivió un proceso de importantes cambios urbanos que hoy día lo llevan a ubicarse como una de las ciudades regionales más importantes de Latinoamérica, con importantes avances en crecimiento y desarrollo económico sostenido en la última década. Incluso, por encima de la media latinoamericana, lo que desencadenó de la mano de políticas y programas públicos, que actualmente el $87 \%$ de los chilenos viva en ciudades. De hecho, de los 6.06 millones de habitantes de la RMS en 2002, 5.87 millones eran de población urbana (Datos INE, 2014; CChC, 2013), lo que abre el debate sobre las serias problemáticas urbanas que hoy aquejan a esta gran urbe (transporte público, parque vehicular, infraestructura vial, contaminación, crecimiento de la mancha urbana, cambios en la geografía metropolitana, etc.).

Encontramos así, que a partir de 1991 y como medida de recuperación posterior al terremoto de 1985, se emprendió el proyecto de renovación urbana del área central de Santiago, con programas de subsidio habitacional y de renovación urbana por parte del Estado Chileno, buscando el mejoramiento, renovación, rehabilitación o remodelación de zonas urbanas (Hidalgo y Arenas, 2011: 5). Así también el desarrollo del Plan de repoblamiento y la construcción de obras públicas (Contreras, 2011), fomentaron la inversión económica privada en el área central de Santiago, sin dejar de lado la propia dinámica natural de crecimiento y desarrollo de la sociedad chilena.

En el marco de estos procesos de cambio y revitalización, tenemos que el centro histórico de Santiago -su triángulo fundacional- posee gran vigencia y vitalidad pues, no sólo concentra las principales funciones e instituciones públicas del país, sino las principales oficinas matrices de instituciones financieras y gerencias de importantes empresas, además de los altos flujos de personas que ahí estudian o trabajan y la gran afluencia turística; aunque ciertas zonas muestran signos de obsolescencia física, funcional y económica en sus inmuebles o el espacio urbano (Green y Soler, 2004: 72,74). A pesar de lo anterior, descubrimos que para el 2012, la Provincia de Santiago concentró el $75.53 \%$ del total de viviendas de la RMS, estimado en 2.1 millones, liderando a su vez la comuna de Santiago el ranking de mayor producción de vivienda en el 
periodo $2002-2012$, con un incremento del $93 \%$, lo que equivale a poco mas de 72,000 viviendas ligadas principalmente a edificios de departamentos (CChC, 2013: 10-12).

¿Los datos anteriores qué implican? Fuertes transformaciones socio-espaciales del centro histórico de Santiago, reflejadas en un continuo proceso de rehabilitación y reciclado no sólo de la vivienda sino de los inmuebles o la renovación de los mismos, que se traduce en la construcción de edificios que rompen en diversos casos con las características de la configuración e imagen urbana del centro (alturas, diseño, materiales), privilegiando la renta por encima de la calidad del paisaje y el bienestar de los habitantes (Hidalgo y Arenas, 2011). Pero ante todo, encontramos un nuevo tipo de habitante atraído por el estilo de vida urbana impulsado en los últimos años y que revalora el potencial de la ubicación, el equipamiento, la infraestructura, el comercio, los servicios y la carga simbólica del Patrimonio urbano, arquitectónico y cultural del centro histórico, junto con las bondades de la cercanía vivienda-trabajo o vivienda-estudios.

Observamos en este último aspecto, que han sido generaciones jóvenes de estratos medios y medios altos (yuppies y dinks) profesionistas y estudiantes los que han llegado al centro histórico, interesados como señalamos, en esa nueva "oferta" inmobiliaria y cultural, por la comodidad del desplazamiento, y por un atractivo estilo de vida urbano (Contreras, 2011: 102) fruto, de los cambios socio-económicos experimentados en los últimos años y como resultado del sostenido crecimiento económico de Chile, el aumento del ingreso per-cápita y los ingresos de los hogares, y el éxito de Santiago como el principal foco articulador de Chile con el resto del mundo (De Mattos, 1999: 40).

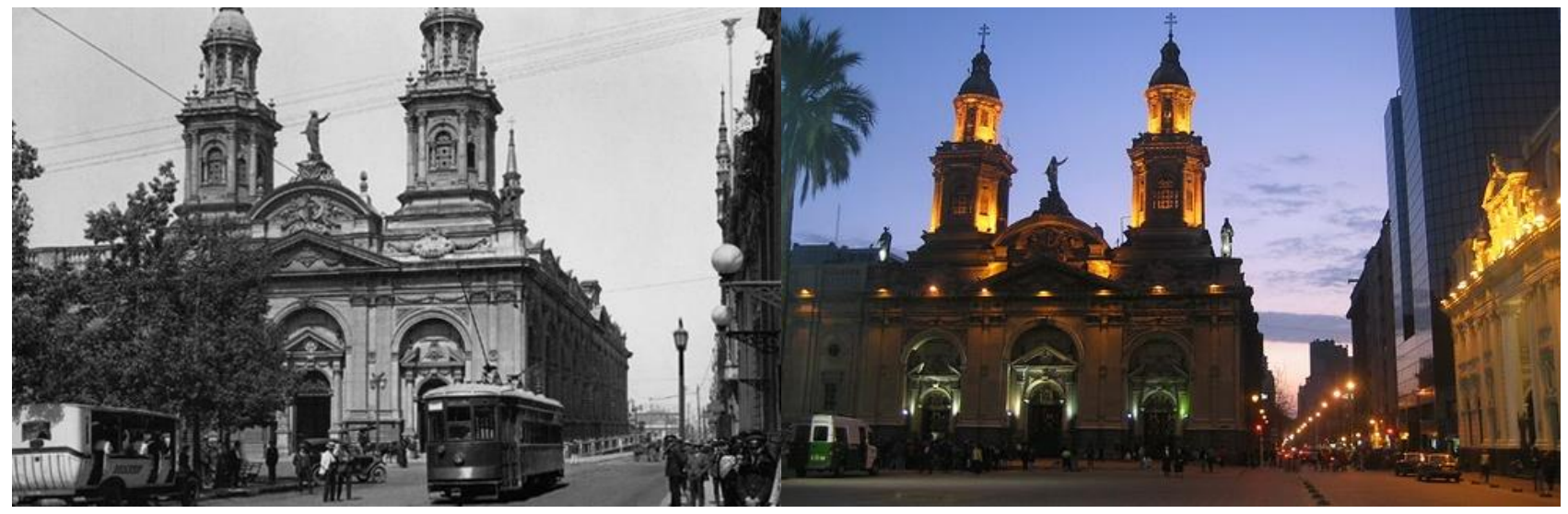

Fig. 2 Catedral de Santiago, Chile (Izq. 1926, Der. 2010)

Fte.: (Izq.) http://www.memoriachilena.cl/602/w3-article-68446.html, (Der.)

http://www.dondeviajar.es/files/2010/12/1-centro-historico-tomada-de-flickr-por-kyle-simourd.jpg

\subsection{El caso Ciudad de México (CDMX).}

La Ciudad de México (Distrito Federal) es la capital de la República Mexicana. La Zona Metropolitana del Valle de México (ZMVM) se integra por las 16 delegaciones del Distrito Federal, 1 municipio de Hidalgo y 59 municipios del Estado de México (SEDESOL, 2012). La ZMVM es la más grande y poblada de Latinoamérica y la 4 cuarta más grande aglomeración urbana del planeta, concentrando una población de 21.17 millones de habitantes, además de que la ONU la ubica en el ranking de las ciudades con sólidos factores de prosperidad (ONU-HABITAT, 2012; ONU, 2014; datos CONAPO).

La Ciudad de México contaba con una población de 471 mil habitantes en 1910, 3.05 millones de habitantes en 1950, 8.83 millones de habitantes en 1980, 8.23 millones de habitantes en 1990, 8.85 millones de habitantes en 2010, estimándose 8.87 millones de habitantes en 2014 , mientras que, la ZMVM contaba con una población de 14.12 millones de habitantes en 1980, 15.56 millones 
de habitantes en 1990, 18.40 millones de habitantes en 2000, 20.12 millones de habitantes en 2010, estimándose 21.17 millones de habitantes en 2014 (Fig. 3). (SEDESOL, 2012; ONU, 2014; datos INEGI y CONAPO).

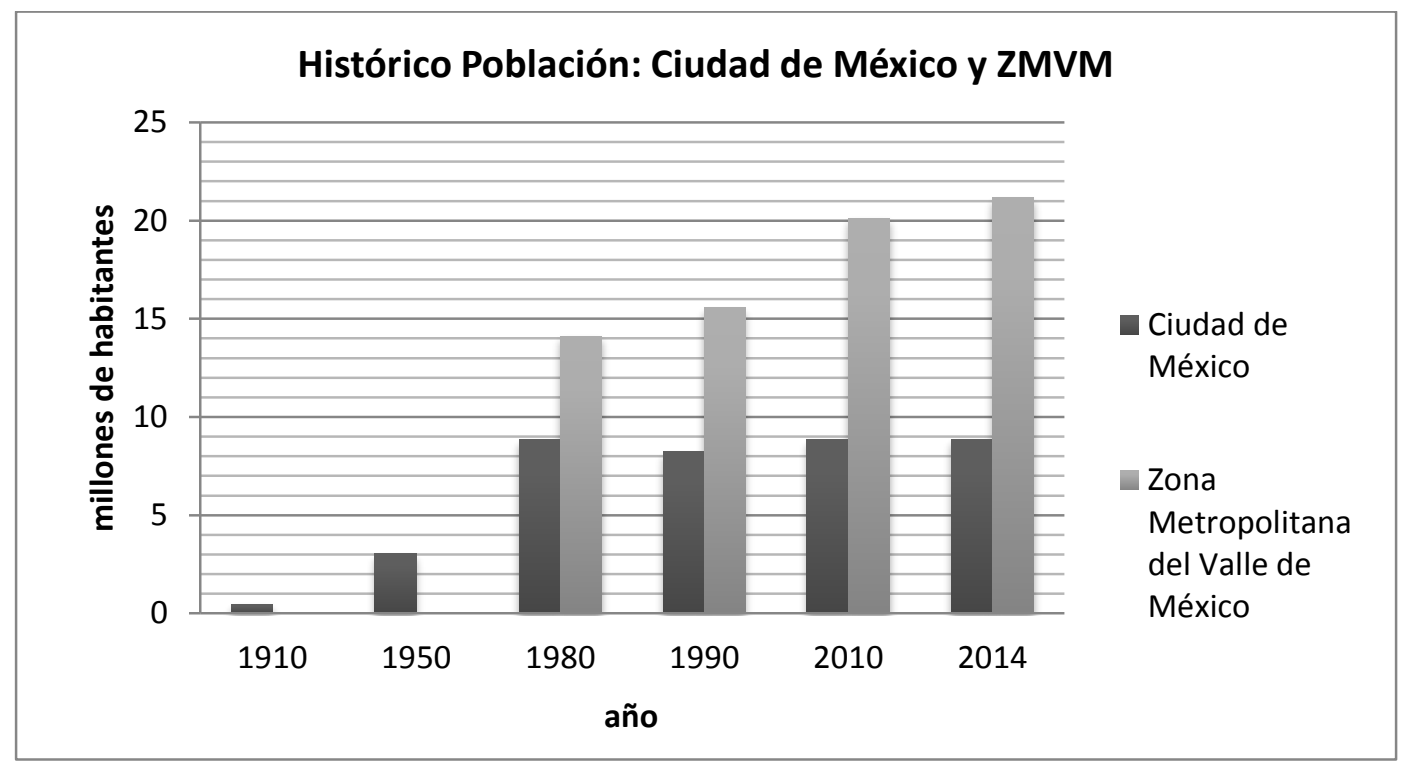

Fig. 3 Cuadro histórico de población para Ciudad de México y la Zona Metropolitana del Valle de México (ZMVM)

Fte.: Elaboración propia con datos de censos INEGI (1910, 1950, 19820, 1990, 2010) y estimaciones CONAPO (2014).

La Ciudad de México experimentó un fuerte proceso de cambio a lo largo del siglo XX, generado por un enorme crecimiento territorial y poblacional, producto del desarrollo económico que vivió el país hacia la primera mitad de ese siglo (Sobrino, 2003: 204), además de ser la ciudad con mayor importancia económica, política y social, generadora de crecimiento a nivel nacional pero que, la llevó a experimentar una expansión urbana desmedida a partir de la segunda mitad del siglo XX y hasta la década de los 80 . Posterior a los sismos de 1985, la ciudad presentó una disminución de población principalmente en el centro histórico, afectado fuertemente en sus inmuebles e infraestructura, sumado a la degradación que venían presentando al igual que el espacio público.

Es a partir de la década de los 90 y posterior a su declaración como Patrimonio Cultural de la Humanidad por la UNESCO en 1987, que comienza el desarrollo de programas tendientes a su rehabilitación, revitalización y repoblamiento. La Ciudad de México es una ciudad expulsora de población pobre pues, aunque continuamente miles de personas llegan a ella en busca de mejores oportunidades, el nivel de vida y el costo de la vivienda resultan costosos para la población de bajos recursos, por lo que tienen que buscar acomodo en los municipios de la zona oriente del Estado de México (integrantes de la ZMVM), en donde encuentran acceso a una vivienda económica y, en muchos casos precaria (Delgadillo, 2013). Este proceso en las últimas décadas ha llevado a este mega crecimiento de la ZMVM y del Estado de México, el más poblado de todo el país con una población de 15.18 millones de habitantes en el 2010 y una estimada de 16.61 millones de habitantes en el 2014 (datos INEGI y CONAPO).

En este contexto, encontramos que las propuestas de rehabilitación y rescate del centro histórico, emprendidas hacia 1991 y que incluían un fideicomiso encargado de la promoción de iniciativas inmobiliarias y el desarrollo de un centro financiero en la zona no se concretaron, principalmente por una fuerte oposición popular (Díaz Parra, 2014: 4) al hecho de que se construyeran oficinas 
y comercios en vez de vivienda popular, además de la fuerte crisis económica que estalla en 1994 (Salinas, 2013: 295), al término de la presidencia de Carlos Salinas de Gortari, principal impulsor de las políticas neoliberales a nivel federal.

Es hasta el año 2000, con Andrés Manuel López Obrador al frente del gobierno capitalino, que se retoma el proyecto con modificaciones, poniéndose en marcha la instalación de varios edificios públicos del gobierno de la ciudad, hoteles y proyectos inmobiliarios de vivienda para residentes de estatus elevado (Díaz Parra, 2014: 4), junto con recursos administrativos (Bando número 2) cuyo objetivo fue: promover la redensificación de un sector de la población mediante la promoción de vivienda de interés social en las delegaciones centrales que presentaban una subutilización de la infraestructura urbana (Salinas, 2013: 296). En este periodo se introduce también el esquema de participación público-privada para la gestión urbana, como fue el caso del Consejo Consultivo para el Rescate del Centro Histórico, presidido por el magnate mexicano Carlos Slim, quien a su vez, dirige su Fundación Centro Histórico para el "rescate" del mismo. Tan sólo entre 2002 y 2004, Slim compró 63 inmuebles en la zona centro y sur poniente del centro histórico para rehabilitarlos y destinarlos a usos comerciales, servicios, vivienda y para sus propias empresas (Delgadillo, 2013: 7, 10).

Si bien a partir de este periodo se ha promovido el repoblamiento del centro histórico, con la promesa de oferta de vivienda social, los estudios indican que en la última década salió más gente de la que llegó, además de que la vivienda desarrollada ha sido en su mayoría para estratos medios y medios-altos (Salinas, 2013; Delgadillo, 2013; Díaz Parra, 2014). Ello además ha representado un encarecimiento de la vivienda para las clases populares y su consecuente expulsión pues, el costo de la vivienda se incrementó entre $150 \%$ y $200 \%$ e incluso más en algunas zonas, al grado de que el valor del metro cuadrado de terreno pasó de 500 USD, a 10,000 USD en ese mismo periodo (Salinas, 2013: 295, 296), teniendo en este 2015 zonas sobre Paseo de la Reforma en las que gracias al desarrollo de exclusivas torres de oficinas, el valor del metro cuadrado de terreno ha pasado de 9,000 USD, a 25,000 USD.

A todo esto, hay que añadir el aumento en la seguridad pública, la rehabilitación del espacio público y su peatonalización en calles específicas (como es el caso de la calle Madero, que recibió el premio Vivir Mejor en la Ciudad del programa ONU-Hábitat); la eliminación o reubicación del comercio ambulante en ciertas zonas, y toda la oferta comercial y de servicios, destinada a los nuevos habitantes (predominantemente generaciones jóvenes de estudiantes, profesionistas, artistas), usuarios de alto estatus y turistas, atraídos además, por la enorme y diversa propuesta artística, gastronómica, arquitectónica, museística y artística que el centro histórico de la Ciudad de México ofrece, lo que es un claro indicador de los cambios socio-espaciales experimentados por ésta en las últimas décadas.

\section{Lugares Heterogéneos: Zonas de Encuentro e Identidad.}

En los ejemplos de las ciudades abordadas, observamos que tanto para el caso de Santiago, como de Ciudad de México, en las últimas dos décadas se dio un fuerte proceso de transformación socio-espacial, que implica más allá de la mera rehabilitación o "rescate" de sus centros históricos y zonas patrimoniales, la irrupción de procesos de gentrificación. Si bien no es posible generalizar las características de este proceso, o hablar de procesos idénticos por la forma, contexto y desarrollo de los mismos, se puede afirmar que la constante es que ambas intervenciones tendieron a captar un nuevo tipo de usuario y de habitante, principalmente generaciones jóvenes, estudiantes y profesionistas, de estratos socio-económicos medios y medios-altos, que ven en ese retorno a la ciudad central, un atractivo estilo de vida urbano en el que convergen estudios, trabajo, vivienda y toda clase de satisfactores (comercio, servicios, cultura, ocio, equipamiento) 
al alcance de la mano, con la riqueza simbólica y patrimonial que ofrece un centro histórico de este tipo.
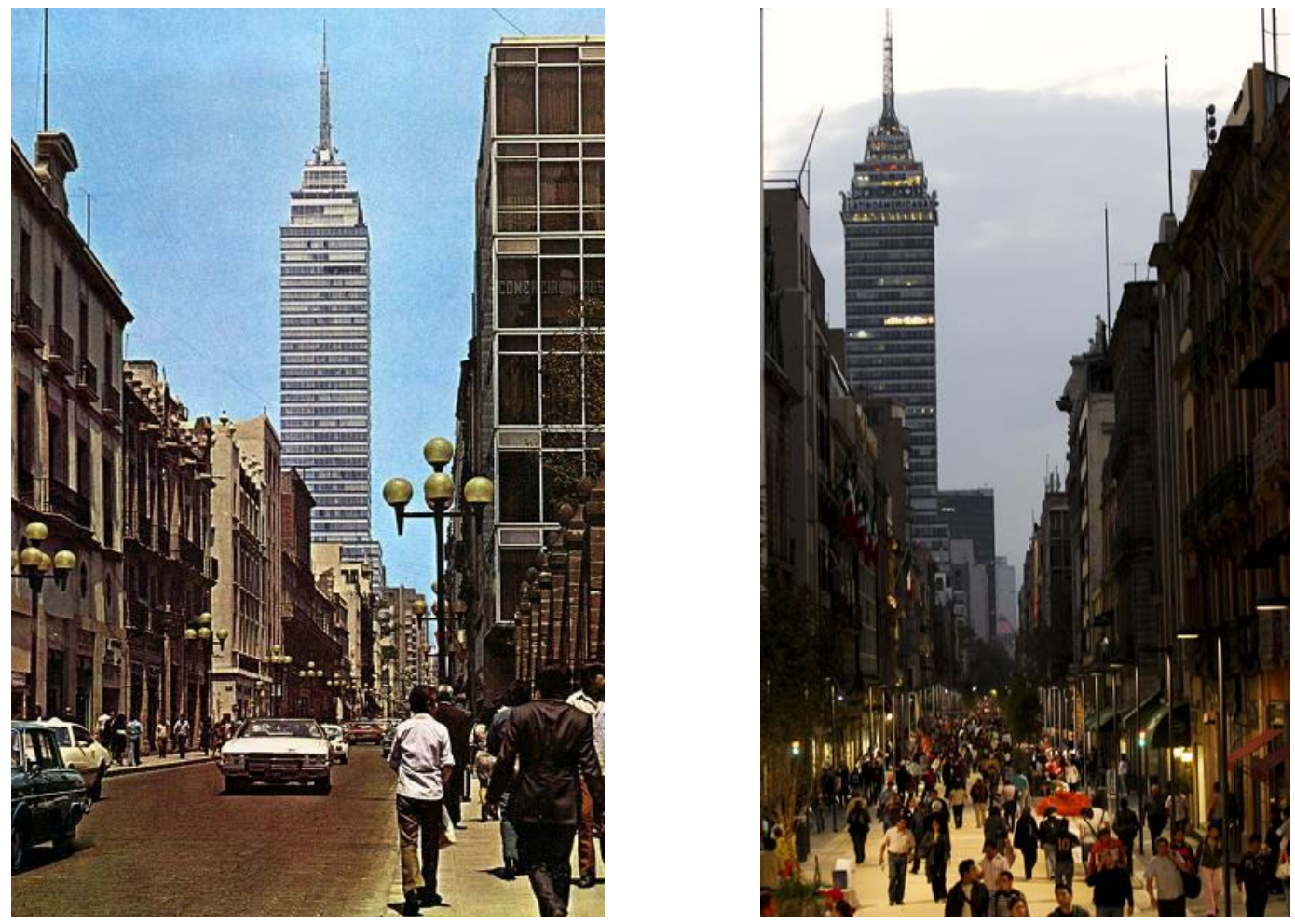

Fig. 4 Calle Madero, CDMX (Izq. 1973, Der. 2015)

Fte.: (Izq.) http://fotos.eluniversal.com.mx/web_img/fotogaleria/ciudad_madero_parteII_10.jpg, (Der.) http://fotos.eluniversal.com.mx/web_img/fotogaleria/010calle-madero2.jpg

Aunque el proceso anterior es la constante en ambas ciudades, hay que aclarar que la gentrificación es puntual y en lugares (calles y cuadras) muy definidos de ambas. No se puede hablar de un fenómeno de gran escala o que la gentrificación abarque todas las zonas que han sido rehabilitadas o intervenidas, lo que no implica que el fenómeno deba verse como un efecto colateral e inherente a la rehabilitación urbana y menos aún como algo bueno y deseable, como sucede con la actual administración de la Delegación Miguel Hidalgo (2012-2015) en Ciudad de México, que anuncia la gentrificación como un "proceso de transformación urbana en el que la población original de un barrio deteriorado se renueva" y como "símbolo de crecimiento económico y mejora de la calidad de vida"2 pues, no olvidemos que el término gentrificación se acuñó para hacer referencia a un problema social y de desigualdad de clase en los barrios (Salinas, 2013: 285).

Vemos cómo coexisten en ambos centros históricos realidades dispares y contrastantes; zonas bien dotadas, revitalizadas y cotizadas, contra zonas degradadas, pobres y obsoletas; una realidad urbana en donde la fragmentación urbana y la segregación están presentes; las zonas tugurizadas siguen existiendo en nuestros centros históricos; la precariedad, la informalidad y la exclusión siguen siendo lacerantes realidades en las ciudades latinoamericanas, zonas de ricos y zonas de pobres, aunque ambos centros fundacionales son quizá los lugares más heterogéneos, identitarios y con mayor apropiación simbólica por parte de sus habitantes. Lugares multiculturales, en donde la carga simbólica de éstos se expresa en su riqueza Patrimonial

2 http://www.miguelhidalgo.gob.mx/sitio2013/que-es-la-gentrificacion/ 
(histórica, urbana, arquitectónica, artística, cultural) que les da esa vitalidad como zonas de encuentro, reunión y de convivencia del espacio público.

\section{Conclusiones}

La heterogeneidad que caracteriza a estos centros históricos de los ejemplos revisados Santiago, Chile y Ciudad de México - es lo que les da riqueza y a la vez, lo que les permite agrupar toda su diversidad la cual, hace posible la convivencia de todos esos actores disímbolos. Los centros históricos de nuestras ciudades requieren de esa diversidad para seguir vivos y vigentes, no se les puede amputar los usos y funciones que tradicionalmente han albergado por siglos pues, sería condenarlos al abandono y la obsolescencia. Las improntas socio-culturales que históricamente han albergado los centros fundacionales de las ciudades latinoamericanas seguirán siendo una constante arraigada en el imaginario colectivo, aunque su espacio urbano se rehabilite y se renueve, aunque sus inmuebles se reciclen.

La gentrificación y elitización de sus espacios existe, es parte ya de su realidad; la turistización y mercantilización de los centros históricos y su Patrimonio es reciente en Latinoamérica pero, es característica de una sociedad globalizada que tiene acceso a mayores ingresos, mejor conectividad y facilidad de movilidad internacional, sin embargo, se debe velar por la conservación del patrimonio urbano-arquitectónico, desde el reconocimiento del barrio y las identidades locales y avanzar hacia una planificación que facilite conductas y estilos de vida más sostenibles (Inzulza y Galleguillos, 2014: 153) respetando el derecho que todos los actores sociales tienen de disfrutar, vivir e identificarse en el espacio público de la ciudad, además de que los residentes locales deben ser los primeros beneficiados de los programas de revitalización y regeneración del espacio urbano, es decir, la gente que en su vida cotidiana los vive, los transita y hace suyos.

Por ello, en este siglo XXI es fundamental promover un urbanismo ciudadano caracterizado por una política urbana centrada en la valoración de la realidad plural y diversa de los barrios y comunidades (Inzulza y Galleguillos, 2014: 153), más allá de una toma de decisiones unilateral que no considera el sentir y la opinión de la población, e impone como única y verdadera la visión de los cuadros técnicos, que en no pocas ocasiones desembocó en propuestas frías, descontextualizadas y ajenas a las necesidades socio-espaciales propias de su momento histórico.

La elitización existe, las áreas gentrificadas son una realidad, presenciamos un escenario que ha sido definido por Inzulza (2012) como latino gentrificación, sin embargo, creemos que en esa mezcla de miles o millones de personas, historias, sucesos, de encuentros sociales que día a día tienen lugar en los espacios públicos de la ciudad, está lo que permite que estos centros históricos no se gentrifiquen a una escala mayor pues, los procesos identitarios que la gente establece con los espacios que vive día a día y la apropiación simbólica que realiza de los mismos en su cotidianidad, favorece que los centros históricos sean lugares cargados de múltiples simbolismos y significados, lugares inscritos en el imaginario de los habitantes, gracias a quienes, los centros históricos de las ciudades latinoamericanas se mantienen vivos, vigentes y a la vez, cambiantes, al igual que las generaciones que los construyen y los hacen suyos. Quede aquí la reflexión y apostemos por nuevos trabajos que abonen al estudio y discusión de los procesos de cambio de las ciudades latinoamericanas. 


\section{Bibliografía}

Borsdorf, Axel; Hidalgo, Rodrigo, "Revitalization and tugurization in the historical centre of Santiago de Chile", en Cities, no. 31, 2012, pp. 96-104, ISSN-0264-2751.

Carrión, Fernando, "Las nuevas tendencias de la urbanización en América Latina", en Carrión, F. (editor), La ciudad construida, urbanismo en América Latina, Ecuador, FLACSO, 2001, pp. 7-24, ISBN-9978-67057-2 http://www.flacso.org.ec/docs/sfcccarrion.pdf (10 de septiembre de 2014)

Cámara Chilena de la Construcción, Análisis y conclusiones resultados preliminares censo 2012. Minuta CTR No. 04, Santiago de Chile, Cámara Chilena de la Construcción, 2013, 43 págs., http://www.cchc.cl/wp-content/uploads/2013/03/Minuta-CTR-N\%C2 \%BA-04-ANALISIS-YCONCLUSIONES-RESULTADOS-PRELIMINARES-CENSO-2012.pdf (07 de octubre de 2014).

Contreras, Yasna, "La recuperación urbana y residencial del centro de Santiago: Nuevos habitantes, cambios socioespaciales significativos", en EURE, vol. 37, no. 112, Santiago de Chile, Pontificia Universidad Católica de Chile, 2011, pp. 89-113, ISSN-0250-7161.

Delgadillo, Víctor, "Ciudad de México, política pública, negocios inmobiliarios, y malestar social", documento de trabajo, Contested Cities - Ciudades en Disputa, 2013, 27 págs., http://www2.aq.upm.es/Departamentos/Urbanismo/blogs/re-hab/wp-content/blogs.dir/9/ files/2013/11/VDelgadillo.pdf (03 de octubre de 2014).

De Mattos, Carlos, "Santiago de Chile, globalización y expansión metropolitana: lo que existía, sigue existiendo", en EURE, vol. XXV, no. 77, Santiago de Chile, Pontificia Universidad Católica de Chile, 1999, pp. 29-56, ISSN-0250-7161.

Díaz-Parra, Iván, "Postfordismo periférico y regreso a la ciudad construida. Discurso y práctica de la regeneración de centros históricos en Iberoamérica", ponencia presentada en el Seminario Internacional Repensar la Metrópoli II. Octubre, México, UAM-PUEM, 2013, 15 págs.

Díaz-Parra, Iván. (2014). "La transformación del centro histórico de la Ciudad de México. ¿Gentrificación o repoblamiento?", ponencia presentada en el XII Coloquio y Trabajos de campo del grupo de geografía urbana. Territorios Inconclusos y sociedades rotas, (AGE), Junio, Madrid - Castilla la Mancha, 2014, 10 págs., http://www.uib.cat/ggu/docs/12col_ggu/Archivos/28.pdf (23 de septiembre de 2014).

García Canclini, Néstor, La Globalización Imaginada, México, Paidós, 2002, 238 págs., ISBN 968-853-434$\mathrm{X}$.

Gómez-Carmona, Gabriel; Villar, Alberto, "La transformación socio-espacial de lugares tradicionales en el contexto metropolitano", ponencia presentada en el Seminario Internacional Repensar la Metrópoli II. Octubre, México, UAM-PUEM, $2013 a$.

Gómez-Carmona, Gabriel; Villar, Alberto, "Apropiación simbólica y reconfiguración identitaria del espacio urbano en Metepec, Estado de México", en Revista Electrónica Nova Scientia, vol. 6 (1), no. 11, México, Universidad De La Salle Bajío, 2013b, pp. 268-290, ISSN-2007-0705, http://nova_scientia.delasalle.edu.mx/numero_11/?lan =es (25 de noviembre de 2013).

Greene, Margarita; Soler, Fernando, "Santiago: De un proceso acelerado de crecimiento a uno de transformaciones", en De Mattos et.al. (editores). Santiago en la globalización: ¿Una nueva ciudad?, Santiago de Chile, Ediciones SUR-EURE, 2004, pp. 47-84.

Hidalgo, Rodrigo; Arenas, Federico, "Negocios inmobiliarios y la transformación metropolitana de Santiago de Chile: Desde la renovación del espacio central hasta la periferia expandida", en Revista Geográfica de América Central, número especial EGAL, II semestre, Costa Rica, Universidad de Costa Rica, Universidad Nacional, Costa Rica, 2011, pp. 1-16, ISSN-2115-2563 http://www.revistas.una.ac.cr /index.php/geografica/article/view/2207 (09 de septiembre de 2014). 
Inzulza, Jorge, "Latino Gentrification? Focusing on physical and socioeconomic patterns of change in Latin American inner cities", en Urban Studies, vol. 10, no. 49, United Kingdom, 2012, pp. 2085-2107, ISSN0042-0980.

Inzulza, Jorge; Galleguillos, Ximena, "Latino gentrificación y polarización: transformaciones socioespaciales en barrios pericentrales y periféricos de Santiago, Chile", en Revista de Geografía Norte Grande, vol. 58, Santiago de Chile, Pontificia Universidad Católica de Chile, 2014, pp. 135-159, ISSN0379-8682.

ONU, World Urbanization Prospects, highlights, New York, United Nations-Department of Economic and Social Affairs, 2014, http://esa.un.org/unpd/wup/Highlights/ WUP2014-Highlights.pdf (07 de octubre de 2014).

ONU-HABITAT, State of the world's cities 2012-2013, prosperity of cities, Nairobi, UN-HABITAT, 2012, http://mirror.unhabitat.org/pmss/listItemDetails.aspx?Publication ID=3387 (07 de octubre de 2014)

Salinas, Luís, "Gentrificación en la ciudad latinoamericana. El caso de Buenos Aires y Ciudad de México", en Geographos, vol. 4, no.44, España, Universidad de Alicante, 2013, pp. 281-305, ISSN-2173-1276 http://web.ua.es/es/revista-geographosgiecryal/documentos/luissalinas.pdf?noCache $=1363271617297$ (10 de agosto de 2013).

SEDESOL, La expansión de las ciudades 1980-2010. México, SEDESOL, 2012.

Sobrino, Jaime, Competitividad de las ciudades en México, México, El Colegio de México, 2003.

Torres, Alma; Hidalgo, Rodrigo, "Los Peruanos en Santiago de Chile: transformaciones urbanas y percepción de los inmigrantes", en Polis, vol. 8, no. 22. Chile, Universidad Bolivariana, 2009, pp. 307326, ISSN-0718-6568 http://www.redalyc.org/articulo.oa?id $=30512211018$ (03 de septiembre de 2014)

Vergara, Carlos, "Gentrificación y renovación urbana. Abordajes conceptuales y expresiones en América Latina", en Anales de Geografía, vol. 33, no. 1, Madrid, Universidad Complutense, 2013, pp. 219-234, ISSN-0211-9803 http://revistas. ucm.es/index.php/AGUC/article/viewFile/43006/40809 (30 de septiembre de 2014).

\section{Referencias electrónicas}

Biblioteca del Congreso Nacional de Chile, Indicadores demográficos.

http://reportescomunales.bcn.cl/2013/index.php/Santiago\#Poblaci.C3.B3n_total_2002_y_proyecci.C3.B3 n_2012_INE (08 de octubre de 2014).

Biblioteca del Congreso Nacional de Chile, Regiones de Chile. http://siit2.bcn.cl/nuestropais/regiones.htm (08 de octubre de 2014).

CONAPO, Proyecciones de Población. http://www.conapo.gob.mx/es/CONAPO /Proyecciones_Datos (13 de Octubre de 2014).

CONAPO, Zonas Metropolitanas de México, 2010. http://www.conapo.gob.mx/es /CONAPO /Zonas_metropolitanas_2010 (10 de octubre de 2014).

INE, Censo de 1940. http://www.ine.cl/canales/usuarios/cedoc_online/censos/pdf /censo_1940.pdf (08 de octubre de 2014).

INE, Censo de 1952. http://www.ine.cl/canales/usuarios/cedoc_online/censos/pdf /censo_1952.pdf (08 de octubre de 2014).

INE, Censo de 1992. http://www.ine.cl/canales/chile_estadistico/demografia_y_vitales/demografia/pdf /censo1992.pdf (08 de octubre de 2014).

INE, Censo 2002. http://www.ine.cl/canales/usuarios/cedoc_online/censos/pdf /censo_2002_publicado_junio_2005.pdf (08 de octubre de 2014). 
INE, Censo de 2002, Volumen I http://www.ine.cl/canales/usuarios/ cedoc_online/censos/pdf/ censo_2002_volumen_I.pdf (08 de octubre de 2014).

INE, Censo de 2002, Volumen II http://www.ine.cl/canales/usuarios/cedoc_online /censos/pdf/censo_2002_volumen_II.pdf (08 de octubre de 2014).

INE, Proyección de Población 2014. http://www.ine.cl/canales/chile_estadistico/ demografia_y_vitales/proyecciones2014/proyecciones-de-poblacion-2014.xIsx (08 de octubre de 2014).

INE, Síntesis Censal 2002. http://www.ine.cl/cd2002/sintesiscensal.pdf (08 de octubre de 2014).

INEGI, Tabulados Básicos. http://www.inegi.org.mx/sistemas/tabuladosbasicos/ tabentidad.aspx?c=33141\&s=est (10 de octubre de 2014$)$. 